Andréia Ramos ${ }^{1}$

Sílvia Rodrigues Jardim²

\section{Lesão Cerebral Traumática e Acidentes de Trabalho: Estudo de Revisão}

\author{
Traumatc Brain Injury and \\ Work-Related Casualty: a Review
}

O objetivo do trabalho foi revisar os achados de pesquisas, nas literaturas nacional e internacional, sobre a associação entre lesão cerebral traumática (LCT) e acidentes de trabalho. Para revisão do assunto utilizou-se como bases de dados o MEDLINE e LILACS do período de 1991 a 2001. Foram encontrados poucos estudos $(n=4)$ investigando a associação entre LCT e acidente de trabalho. Estes apresentavam limitações metodológicas, tais como utilização de bancos de dados secundários, a caracterização da amostra e os critérios utilizados para definir LCT. Percebe-se uma preocupação com os estudos sobre reabilitação e gastos dos sistemas de saúde com este tipo de lesão - e suas seqüelas -, mas pouca pesquisa envolvendo a discussão da inter-relação da organização do trabalho com a incidência de LCT.

Palavras-chave: lesão cerebral traumática, traumatismo crânio-encefálico, acidente de trabalho, saúde ocupacional.

The aim of this study was to review the research findings both in nationwide and worldwide literature as regards the connection between traumatic brain injury (TBI) and work-related casualty. In order to carry out a review of the issue, database such as the MEDLINE and LILACS were used between 1991 and 2001. Few studies were found (n=4) which investigated the relationship between traumatic brain injury and work-related casualty. These presented methodological limitations such as the use of secondary database and different criteria to define the TBI. Some concern is observed regarding the studies on rehabilitation and on the costs for the health system owing to this kind of damage - and its sequels - but little research comprising the interrelationship between work organization and $T B I$ incidence is found.

Keywords: traumatic brain injury, head injury, work-related casualty, occupational health. 


\section{Introdução}

Existem diversas classificações para as lesões cerebrais traumáticas. Elas podem ser classificadas como penetrantes ou fechadas, dependendo da integridade das meninges após o trauma. As lesões fechadas representam a maioria das lesões cranianas. A principal causa das lesões penetrantes são os ferimentos por projéteis de armas de fogo, que produzem lesões características e podem ser complicadas por hemorragia e infecção (Jorge \& Robinson, 1998).

As lesões também podem ser classificadas como primárias ou secundárias. As primárias são produzidas por mecanismos diretamente associados ao evento, ou seja, no momento do trauma. A conseqüência pode ser uma lesão focal - como contusão, hemorragia intracerebral ou epidural - ou difusa - lesão medular, isquemia difusa. As lesões secundárias são aquelas decorrentes de processos patológicos que começam no momento do dano, mas que se estendem por um período variável após o episódio traumático (Jorge \& Robinson, 1998).

Uma terceira classificação é aquela baseada na extensão e na localização das mudanças neuropatológicas. Neste caso, as lesões podem ser focais ou difusas.

Embora os critérios classificatórios existam para tentar facilitar o diagnóstico dessas lesões, verifica-se que suas utilizações em pesquisas constituem um fator que, normalmente, confunde mais do que esclarece, gerando problemas de ordem metodológica. Uma das principais dificuldades encontradas é a impossibilidade de se realizar análises comparativas entre pesquisas epidemiológicas sobre lesões cerebrais traumáticas, uma vez que os diagnósticos selecionados para determinar a inclusão da população do estudo não são uniformes (Kraus \& Sovenson, 1994). Dentre as dificuldades constatadas podemos citar a utilização de diferentes referenciais para morbidade e mortalidade, a definição operacional de LCT adotada na pesquisa (que tipo de lesão está sendo considerada: encefálicas, crânio-hencefálicas) e a inclusão da população da pesquisa segundo a gravidade da lesão (incluir apenas lesões graves deixando de fora as leves).

Mesmo que essas limitações sejam apontadas em estudos sobre lesão cerebral traumática de uma forma geral, acreditamos que possam ser extrapoladas quando se discute uma pesquisa envolvendo LCT relacionada ao trabalho.
Os dados epidemiológicos revelam que adolescentes e adultos jovens, seguidos por indivíduos com idade acima de 65 anos, são as populações mais atingidas pelas LCTs (Klauber, Barret-Connor \& Marshall, 1981).

Os homens apresentam uma freqüência duas ou três vezes maior do que as mulheres (Klauber, Barret-Connor \& Marshall, 1981).

A condição socioeconômica inferior é outro fator de risco para LCT. Contudo, o maior fator de risco isolado é o abuso e a dependência de substâncias psicoativas. Aproximadamente um terço dos pacientes com LCT apresenta distúrbio de abuso de substância anterior ao trauma e mais de 50\% estavam intoxicados no momento da lesão (Miller, 1994).

Acidentes envolvendo veículos motorizados, inclusive atropelamentos, são a causa mais freqüente de lesão, particularmente nos pacientes mais jovens (Machado \& Gómez, 1994). As quedas são a segunda causa mais prevalente de lesão craniana, especialmente entre os pacientes mais velhos (Klauber, BarretConnor \& Marshall, 1981). Os assaltos, os acidentes esportivos e de recreação são outras causas importantes de lesão craniana (Machado \& Gómez, 1994). Cerca de 80\% das lesões cerebrais que levam as pessoas a serem hospitalizadas são consideradas leves, $10 \%$ são moderadas e $10 \%$ são graves (Klauber, Barret-Connor \& Marshall, 1981).

$A$ relação entre a $L C T$ e uma variedade de distúrbios neuropsiquiátricos tem sido relatada por muitos anos na literatura médica (Jorge \& Robinson, 1998; Lishman, 1968). No entanto, poucos estudos têm examinado a prevalência de LCT em acidentes de trabatho. Questões como a prevalência de LCT nos locais de trabalho, a notificação desse tipo de patologia como decorrente de acidente de trabalho, as variáveis da organização do trabalho associadas à ocorrência desta patologia, a evolução do quadro clínico e a influência da LCT na evolução longitudinal dos danos físicos e comportamentais pós-lesão são ainda pouco exploradas.

Como já mencionado acima, por ora, interessa-nos abordar a relação entre LCT e acidente de trabalho revendo como este tema é trabalhado atualmente nas literaturas brasileira e internacional. Para a revisão do assunto, foram pesquisados os seguintes bancos de dados: MEDLINE e LILACS, no período de 1991-2001. Na busca, utilizaram-se como palavras-chave: trabalho, trauma 
cerebral, lesão cerebral, traumatismo crânioencefálico, acidente de trabalho, ocupacional em suas possíveis combinações.

\section{Lesão cerebral traumáti- ca e acidente de trabalho: os dados da literatura}

Dentre a bibliografia pesquisada, foram encontrados trinta e quatro artigos que exploravam o tema $\mathrm{LCT}$, porém apenas quatro discutiram a relação entre LCT e acidente de trabalho. Somente três artigos foram analisados por ser um deles em idioma russo, tendo os autores acesso apenas ao resumo deste.

Embora os trinta artigos restantes não tenham sido objeto de nossa análise, consideramos importante destacar alguns dados que nos pareceram significativos para o tema abordado neste trabalho. Dezesseis deles $(56,66 \%)$ discutiram processos e estratégias de reabilitação ao trabalho pós-LCT. Verificou-se que tais estudos não tinham como proposta caracterizar a causa da LCT, de forma que não havia dados que a relacionasse com a ocupação. Eles apontaram para a necessidade de programas específicos de reabilitação ao trabalho, considerando, especificamente, as dificuldades para o desempenho das atividades laborativas decorrentes dos déficits cognitivos pós-lesão.

Treze $(43,34 \%)$ dos trinta artigos tiveram como objeto principal da análise os custos para o sistema de saúde (assistência e previdência), público e privado, dos afastamentos do trabalho devido a lesões traumáticas. Também nesses estudos foram consideradas LCTs relacionadas ou não à ocupação.

Considerando os trabalhos selecionados nesta revisão (1991-2001), observa-se que a relação entre $\mathrm{LCT}$ e acidente de trabalho não é estudada por todos pesquisadores de forma direta. Ou seja, algumas pesquisas investigam essa relação estudando-a dentro de um contexto mais amplo em que se analisam outros fatores associados ao evento. É o caso das pesquisas desenvolvidas por Menard (1996) e Alexander et al. (1999).

O estudo realizado por Menard (1996) objetivou determinar quais tipos de lesões traumáticas ocupacionais estavam associadas à incapacidade prolongada. Para obter seus dados, o autor recorreu ao banco de dados de sistemas de concessão de benefícios. Os resultados encontrados evidenciaram que a incapacidade tinha uma relação de dependência com a gravidade da lesão e que as LCTs estavam associadas à maior incapacidade ocupacional. A discussão do autor envolve a análise de que o tratamento das lesões traumáticas (ocupacionais ou não) e as políticas e as decisões médico-legais acerca delas são baseadas em critérios que as definem como "incapacidades típicas", de modo que se torna necessário uma documentação mais precisa, tanto nos sistemas de benefícios quanto naqueles que se prestam a outros objetivos (Menard, 1996).

Uma documentação mais precisa, segundo Menard, deverá incluir uma classificação mais detalhada da causa, do tipo e da gravidade da lesão e da associação - e persistência desta - da ocupação com a lesão. O estudo de Menard discutiu a questão dos critérios utilizados para identificar esta patologia nos bancos de dados dos sistemas de benefícios previdenciários.

Alexander et al. (1999) conduziram um estudo de comparação entre lesões fatais e não fatais graves relacionadas ao trabalho em trabalhadores do estado de Washington utilizando como banco de dados o sistema de compensação de gastos com internações de trabalhadores apresentando lesões não fatais. As lesões não fatais graves foram caracterizadas como sendo aquelas que resultavam em grave incapacidade ou que requeriam cuidados médicos intensivos. Um dos critérios utilizados pelos pesquisadores para caracterizar uma lesão grave foi a presença de lesão cerebral ou na coluna vertebral, considerando que esses tipos de lesões possuem um alto potencial para gerar incapacidade grave por longo tempo. Os pesquisadores incluíram todas as lesões cerebrais classificadas pela CID-9 - CID 800, 803, 804, 850-854, excluindo concussão sem perda de consciência - e o diagnóstico de uma lesão de coluna vertebral - CID 806, 952 (Alexander, Fraklin \& Fulton-Kehoe, 1999).

Um dos resultados encontrados pela pesquisa foi o de que, no período de 1991 a 1995 , quatrocentos trabalhadores foram internados por apresentarem lesão cerebral ou na coluna vertebral.

Embora esse estudo tenha apresentado importantes dados que diferenciam as lesões fatais das não fatais naquela população como, por exemplo, o achado de que as lesões fatais foram mais freqüentes em traba- 
Ihadores mais velhos -, o fato é que não houve uma preocupação em se estudar a relação direta entre LCT e acidente de trabalho. Não sendo essa a proposta principal deste estudo, não foi possível realizar um cruzamento das variáveis que permitisse caracterizar melhor as LCTs.

O único estudo que analisou diretamente esta associação foi o desenvolvido por Heyer \& Franklin, 1994 (Heijer \& Franklin, 1990). Nessa pesquisa, o objetivo foi identificar casos de LCT e descrever a incidência considerando as variáveis de idade, gênero e classificação da indústria onde ocorreu. Esse estudo utilizou como banco de dados o sistema de registro do Departamento de Trabalho e Indústrias do Estado de Washington (EUA) no período de 01 de janeiro de 1988 a 31 de dezembro de 1990. Foi considerado caso de LCT aquele que, além de causar trauma na região craniana, havia recebido registro de alguma das seguintes situações: evidência clínica ou de imagem (exame de imagem) de lesão cerebral, um diagnóstico médico de concussão ou uma descrição clara de diminuição e/ou alteração do nível de consciência.

A utilização de diferentes variáveis do banco de dados foi realizada com o objetivo de analisar a especificidade de cada uma delas, isoladamente e em conjunto. Estas variáveis foram: (1) dados do departamento: idade, gênero, natureza do código da lesão-Z16.2 (American National Standards Institute) e o código de classificação das indústrias (Washington Industrial Classification); (2) dados de pagamento dos serviços médicos prestados ao trabalhador lesionado: código utilizado pelo hospital (Código Internacional de
Doenças - CID, versão 9), grupo de código de diagnóstico relacionado (DRG) e o tempo de hospitalização.

Os autores identificaram trezentos e um casos de LCT, com declínio do número de hospitalizações em 1990. A hipótese para esta redução, segundo os pesquisadores, é de que houve uma drástica diminuição no número de internações de apenas um dia, que foram significativas nos anos anteriores pesquisados.

Foram três as principais causas de trauma cerebral: quedas $(48,5 \%)$, golpes por objetos $(26 \%)$, acidentes com veículos motores $(18,3 \%)$.

Os sete tipos de indústrias com maior número de ocorrência de LCT (seis ou mais) responderam por $1 / 3(101 / 301)$ de todos os casos. As indústrias com alto risco relativo (RR) foram: atividades de derrubada, corte e transporte de madeira (36.7), atividades em telhado (17.1), coleta de lixo (13.5), construção de estradas e rodovias (12.4), atividade de motorista (10.0), atividades em fazendas de gado leiteiro (8.9), carpintaria de interiores (8.8) e atividades com estruturas de madeiras na construção de edifícios (7.2).

A incidência anual de hospitalização por trauma cerebral relacionado ao tipo de ocupação foi de 9,4 casos/100.000 trabalhadores em atividade de horário integral. A relação homem/mulher foi de 6,3:1. Esta relação difere da encontrada em estudos com população geral, cujos valores são de 2 a 3:1. Segundo os autores, essa discordância pode ser explicada pelas diferentes distribuições de fatores de riscos ocupacionais e não ocupacionais. Outra explicação seria as diferenças nas populações subnotificadas.

Os dados dos artigos analisados estão dispostos no Quadro 1. QUADRO 1

\begin{tabular}{|l|l|l|l|}
\hline \multicolumn{1}{|c|}{ Estudo } & \multicolumn{1}{|c|}{ População do Estudo } & \multicolumn{1}{c|}{ Desenho de Pesquisa } & \multicolumn{1}{c|}{ Resultados } \\
\hline $\begin{array}{l}\text { Heyer \& Franklin, } \\
1994 \\
\text { (EUA) }\end{array}$ & $\begin{array}{l}\text { Trabalhadores de } \\
\text { indústrias }\end{array}$ & $\begin{array}{l}\text { Análise de dados de benefícios } \\
\text { concedidos (estudo retrospectivo) }\end{array}$ & $\begin{array}{l}\text { Foram identificados 301 } \\
\text { casos de LCT no período } \\
\text { de 3 anos. Um terço de } \\
\text { todas as lesões estavam } \\
\text { concentradas em 7 tipos } \\
\text { específicos de indústrias }\end{array}$ \\
\hline $\begin{array}{l}\text { Menard, 1996 } \\
\text { (Canáda) }\end{array}$ & $\begin{array}{l}\text { Trabalhadores de } \\
\text { Vancouver }\end{array}$ & $\begin{array}{l}\text { Análise de dados de sistema de } \\
\text { benefícios (estudo retrospectivo) }\end{array}$ & $\begin{array}{l}\text { A LCT está associada com } \\
\text { maior incapacidade ao } \\
\text { trabalho }\end{array}$ \\
\hline $\begin{array}{l}\text { Alexander et al., } \\
1999 \\
\text { (EUA) }\end{array}$ & $\begin{array}{l}\text { Trabalhadores hospitali- } \\
\text { zados com lesão } \\
\text { traumática relacionada } \\
\text { ao trabalho }\end{array}$ & $\begin{array}{l}\text { Análise de dados de compensa- } \\
\text { ção com trabalhadores acometi- } \\
\text { dos por lesões traumáticas no } \\
\text { estado de Washington no } \\
\text { período de 1991-1995 }\end{array}$ & $\begin{array}{l}\text { Foram identificados 400 } \\
\text { casos de lesão cerebral ou } \\
\text { de coluna vertebral. }\end{array}$ \\
\hline
\end{tabular}




\section{Discussão e conclusão}

Os resultados dos estudos analisados indicam que a ocorrência de LCT como acidente de trabalho ainda é pouco explorada, enquanto associação direta, o que pode ser conseqüência das limitações metodológicas dos poucos estudos até agora realizados.

Todos os estudos analisados foram retrospectivos, utilizando dados secundários. O uso exclusivo do desenho retrospectivo - neste caso - impõe limites. A utilização de dados secundários também mostra limitações pela qualidade freqüentemente comprometida dos registros e pela ausência de registros sistemáticos ao longo do tempo.

Outro aspecto a ser ressaltado diz respeito à forma como as populações dos estudos analisados foram definidas. Os estudos incluíram apenas trabalhadores afastados, não havendo informações sobre aqueles em atividade. As pesquisas envolvendo trabalhadores ativos limitaram-se a discutir processos de reabilitação e não especificavam se a LCT tinha relação com acidente de trabalho. No entanto, estes dados referem-se aos trinta artigos que não foram utilizados neste trabalho.

Embora a escassez de estudos nesta área possa indicar a existência de número reduzido de trabalhadores com LCT ou até a pequena importância do problema na população, vale ressaltar que não é essa a realidade quando se constata o número elevado de estudos envolvendo os custos de afastamento do trabalho e as propostas de reabilitação para o trabalho dos indivíduos com LCT.

No Brasil, os dados sobre o assunto são ainda mais precários, mesmo quando se examina o banco de dados de órgãos oficiais. $O$ banco de dados do MPAS mostra claramente esta realidade ao limitar seus dados a informações isoladas e pouco detalhadas (Brasil, 2002).

Percebe-se uma preocupação com os estudos sobre reabilitação e sobre os gastos dos sistemas de saúde com este tipo de lesão - e suas seqüelas -, mas há pouca pesquisa envolvendo a discussão da inter-relação da organização do trabalho com a incidência de
LCT. Acreditamos que este tipo de questão é anterior àquela sobre prevenção e reabilitação e, ao examinarmos a produção científica sobre o assunto, fica evidente a necessidade de que tais estudos se desenvolvam.

Uma discussão mais ampla implica, também, em reconhecer que o problema não se esgota nos fatos de que existam poucos estudos sobre o assunto, de que a maioria dos bancos de dados existentes possui sérias limitações ou ainda de que os órgãos oficiais não fornecem atenção necessária ao problema. Trata-se, sim, de perceber que os trabathadores vítimas de LCT por acidentes de trabalho passam por um longo processo de sofrimento, que não é só físico - representado por seqüelas - mas também psicológico. A experiência de um corpo com limitações, do afastamento do trabalho provocado pelo fato de não ser mais considerado apto para exercê-lo, a dificuldade de se reinserir no mercado de trabalho constituem, para uma grande parcela de trabalhadores acometidos por LCT, a nova realidade na qual são inseridos. É a experiência de se defrontar com o fato de não ser mais aquele mesmo indivíduo/trabalhador.

Esses trabalhadores constituem uma subpopulação cujas medidas de intervenção nas condições e na organização do trabalho podem modificar os números apresentados em estudos já realizados. Essa subpopulação constitui, também, um grupo de risco para a exclusão social, sem condições de reinserção no trabalho.

Essas questões remetem a uma dimensão que não é apenas a do individual, do psicológico, mas do social e do coletivo, aquela na qual milhares de trabalhadores estão inseridos e submetidos. E se é do campo do social, do coletivo, significa que o campo da saúde do trabalhador tem um compromisso com a produção de conhecimento sobre o tema que permita construir um saber através do qual a relação acidente de trabaIho-lesão cerebral traumática não seja apenas uma preocupação em reabilitar quem já adoeceu. Ela deve ser antecedida pela discussão das possíveis mudanças na organização do trabalho que gera os eventos traumáticos.

\section{Referências Bibliográficas}

\author{
ALEXANDER, B. H.; FRANKLIN, G. M.; \& \\ FULTON-KEHOE, D. Comparison of fatal \\ and severe nonfatal traumatic work-
}

related injuries in Washington State.

Am. J. Ind. Medicine 1999; 36: 317-

325. 
HEYER, N. J.; \& FRANKLIN, G. M. Workrelated traumatic brain injury, in Washington State, 1988 through 1990. Am. J. Public Health 1990; 84: 1106-1 109.

JORGE, R.; \& ROBINSON, R. Distúrbios do Humor após Lesão Cerebral Traumática. Rumos em Psiquiatria. In: Educação Médica Contínua. Fascículo II. Rio de Janeiro: EPUC Editora de Publicações Científicas; 1998. pp. 2-16.

KLAUBER, M. R.; BARRET-CONNOR, E.; \& MARSHALL, L. F. The epidemiology of head injury: a prospective study of an entire community: San Diego County, California, 1978. Am. J. Epidemiology 1981; 113: 500-509.

KRAUS, J. F.; \& SORENSON, S. B. Epidemiology. In: SILVER, J. M.; YUDOFSKY, S. C.; \& Hales, R. E. Neuropsychiatry of Traumatic Brain Injury. Washington, DC: American Psychiatric Press; 1994. pp. 3-42.
LISHMAN, W. A. Brain damage in relation to psychiatric disability after head injury. British J of Psychiatry 1968; 114: 373-410.

MACHADO, J. M. H.; \& GÓMEZ, C. M. Acidentes de trabalho: uma expressão da violência social. Caderno de Saúde Pública 1994; 10 (Sup. 1): 74-87.

MENARD, M. R. Comparison of disability behavior after different sites and types in a workers' compensation population. J. Occup. Environ. Medicine 1996; 38 (11): 1161-1170.

MILLER, N. S. Alcohol and drug disorders. In: SILVER, J. M.; YUDOFSKY, S. C.; \& HALES, R. E. Neuropsychiatry of Traumatic Brain Injury. Washington, DC: American Psychiatric Press, 1994, pp. 471-498.

BRASIL. Ministério da Previdência e Assistência Social. Acidentes do Brasil. Trabalho, 1998-2000. Disponível em URL: http:// www.mpas.gov.br [2002] 